\title{
Frontières
}

\section{Quand la vie donne envie d'euthanasie}

\section{Claire Morissette}

Volume 20, numéro 1, automne 2007

La « bonne mort»

URI : https://id.erudit.org/iderudit/017959ar

DOI : https://doi.org/10.7202/017959ar

Aller au sommaire du numéro

Éditeur(s)

Université du Québec à Montréal

ISSN

1180-3479 (imprimé)

1916-0976 (numérique)

Découvrir la revue

Citer cet article

Morissette, C. (2007). Quand la vie donne envie d'euthanasie. Frontières, 20(1), 103-104. https://doi.org/10.7202/017959ar d'utilisation que vous pouvez consulter en ligne.

https://apropos.erudit.org/fr/usagers/politique-dutilisation/ 


\section{QUAND LA VIE DONNE ENVIE D'EUTHANASIE}

\author{
Claire Morissette, \\ fondatrice de Cyclo Nord-Sud, \\ lauréate du prix Thérèse-Daviau 2007.
}

Claire Morissette se qualifiait de «vélorutionnaire». Grande environnementaliste, elle a œuvré pour développer l'usage de la bicyclette avec le mouvement "Le monde à bicyclette", à qui l'on doit l'aménagement de centaines de kilomètres de pistes sur l'île de Montréal. Elle a aussi consacré les sept dernières années de sa vie à mettre sur pied le projet Cyclo Nord-Sud qui a acheminé 20000 vélos vers 13 pays. À titre posthume, elle a reçu le prix ThérèseDaviau 2007, décerné à des citoyennes ayant contribué à l'amélioration de la qualité de vie de la métropole du Québec.

Ce texte, écrit en juin 2007, n'était pas destiné à être publié, ce qu'elle a signalé à la députée Francine Lalonde ${ }^{1}$, en lui envoyant une copie par courriel. Madame Lalonde a voulu prendre contact avec elle récemment pour apprendre par son ami qu'elle était morte le 20 juillet. Celui-ci a autorisé la publication de ce texte troublant.

J'ai frôlé la mort de près à l'hiver 20042005, à la suite d'un cancer du sein métastasé aux os et au foie. J'ai aussi vu mon père décéder d'un cancer généralisé après avoir parcouru le curriculum médical jusqu'à la fin. Voici ce que j'en ai appris.

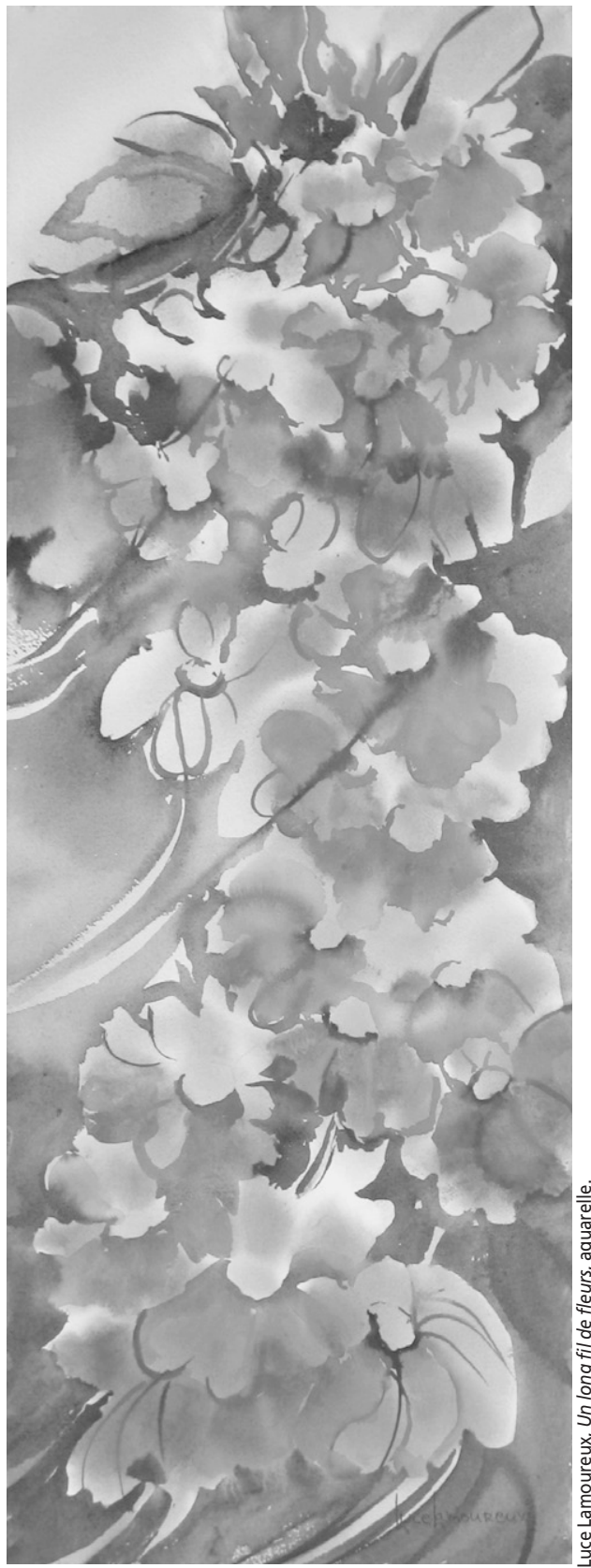

\section{LA DOULEUR}

Souffrir ressemble beaucoup à grelotter. C'est un recroquevillement, une contraction de tout le corps, de la racine des cheveux jusqu'aux pieds. Ça fait MAL!!! Et ça fait mal constamment. Ce grelottement absorbe toutes vos forces, toute votre attention, c'est épuisant. Pensez-y: supporteriez-vous de grelotter dix jours, vingt jours, deux mois, des années?

\section{LE SOULAGEMENT}

Vient la piqûre de morphine. C'est comme une vague de chaleur qui nous délivre du grelottement, une somptueuse relaxation sur une plage étale. "Merci!!! Ah! merci!!! Ça fait tellement de bien!!! » Mais attention, il faut rester bien sage car si vous ouvrez les yeux, c'est le vertige, le décor bascule, la nausée vous prend et peut vous mener au vomissement. Autre désagrément: la constipation vous barre le ventre, vous torture l'anus. Et le répit est de courte durée. Au bout de 3 heures, ce serait le temps d'une autre piqûre mais $\frac{.}{\omega}$ l'infirmière doit attendre 4 heures... et on se remet à grelotter. Et puis il y a l'habituation, il faut augmenter les doses, on devient halluciné, confus, à moitié idiot (j'ai vu mon père dans cet état).

\section{DISGRÂCE ET PESTILENCE}

Pendant ce temps, le corps se vide. Plus d'appétit, ni d'exercice, vous fondez. Dans le miroir, vous êtes un squelette comme ceux des camps de concentration (sans 
exagérer), plus de fesses pour s'asseoir, vos seins sont vidés, aucun confort avec ces genoux cagneux, sauf avec un coussin entre les jambes. La peau ratatine et fait des plis partout. Une disgrâce totale. Pire: à cause de la médication, vos urines, selles, flatulences, haleine, vomissements ont une odeur de fin du monde, que vous imposez à vos aidants, une profonde humiliation. Si vous devez déféquer au lit, en bassine sèche, la puanteur est indescriptible et c'est quelqu'un d'autre qui vous essuie ensuite les fesses, une encore plus grande profondeur d'humiliation.

\section{L'ÉNERGIE}

C'est la panne sèche. Il faut s'y prendre en trois sessions pour laver une petite vaisselle, car ce travail est trop cardio!!! Incapable de faire les repas, le ménage, un travail quelconque. Au début, on lit, on regarde des DVD, on essaie de s'adapter et d'en profiter autrement. Puis, lire devient trop fatigant. On n'aspire plus qu'à sombrer dans le sommeil, l'inconscience et pourquoi pas, le néant.

\section{L'ENTOURAGE}

Tout le monde a de la peine. Ils sont là, font leur possible, assument mal leur impuissance, vont se cacher pour pleurer, même si on essaie de faire régner une atmosphère plutôt matter of fact. Leur chagrin fait peine à voir.

\section{MOURIR DE FAIM OU DE SOIF ?}

Une fois ses moyens d'intervention épuisés, la médecine conventionnelle vous laisse alors vous dégrader de façon «naturelle»: les organes faiblissent et vous découvrez d'autres douleurs, d'autres écœurements. La morphine vous rend à demi-idiot et vous n'êtes même plus réellement présent. Au bout du compte, la médecine vous laisse mourir de faim ou de soif. Si vous acceptez le soluté hydratant, vous mourrez de faim, ce qui peut prendre de 30 à 90 jours. Si vous refusez d'être hydraté, vous mourrez de soif en 10 à 20 jours, dans un état de prostration que je n'ose pas imaginer. Pendant tout ce temps, les gens défilent à votre chevet et vivent un chagrin aigü et prolongé.

\section{EN TERMINER SOI-MÊME}

$\mathrm{Si}$ on cherche, on trouve assez facilement des guides analysant toutes les formes de suicide, selon leur efficacité (on ne veut surtout pas manquer son coup) et leur aspect moins traumatisant pour l'entourage. Ici, il faut agir assez vite, tandis qu'on est encore capable de poser l'acte final, ce qui demande un courage d'une qualité spéciale. Il y a aussi la difficulté de ne pas incriminer ses proches. Comment arriver à avoir quelqu'un qui vous tienne la main au dernier moment, sans qu'il ou elle soit accusée de meurtre. Vous rendez-vous compte de la cruauté de ce dilemme... Il faut surveiller tout, jusqu'aux empreintes digitales!

JE VAIS MOURIR, ET JE LE SAIS.

VOUS AUSSI ALLEZ MOURIR

\section{UN JOUR, LE SAVEZ-VOUS?}

\section{LA MORT, C'EST AUSSI ÇA LA VIE}

Je vais mourir, et je le sais. Vous aussi allez mourir un jour, le savez-vous? Les gens qui clament «La vie, la vie!» semblent souvent vouloir absolument éviter un regard sur la mort, ultime étape de la vie.

La mort est incontournable, et s'y préparer est sage. Mes recherches et lectures sur la mort, loin de m'horrifier, m'ont apporté beaucoup de paix intérieure. On peut réussir sa mort comme on réussit sa vie. Tirer sa révérence en se sentant comblé. Tous ceux qui l'ont frôlée de très près en parlent comme d'une expérience agréable. Il n'y a que deux façons de l'aborder: avec horreur et panique ou avec courage et curiosité. Je cultive la deuxième, bien que je sache ne pas être à l'abri de la première.

\section{LA SAGESSE DU VIEILLISSEMENT}

Il y a une grande sagesse dans le corps vieillissant: la fatigue, la douleur, la dégénérescence font de la mort une amie, une délivrance, une bénédiction. N'est-ce pas magnifique??? Je suis d'accord qu'une mort trop hâtive peut être ressentie comme un scandale et une tragédie, mais lorsqu'on est sur l'autre versant de la vie, elle en vient à offrir un tout autre visage.

\section{SE PRÉPARER À LA MORT}

Pour moi, cela a impliqué de faire le deuil de tout ce qui m'est cher: mon amoureux, mes frères, sœur, neveux et nièces, mes amies, mes œuvres, ma maison, mes petits plaisirs, des grandes choses et des petites «bébelles», TOUT, TOUT me sera arraché! Et j’ai pleuré pendant environ un mois.

Ensuite? Pleurer, c'est ennuyant, et on s'en lasse. Après avoir bien pleuré, j'ai ressenti une paix, le fameux détachement. Et je suis « revenue» dans la vie, en réalisant à quel point tout m'est donné, chaque minute est un bonus que je savoure probablement plus que vous (on gage?). Mais la mort pour moi est enfin dédramatisée.

\section{OUI À L'EUTHANASIE}

Grelottement, nausée, décharnement, épuisement, écœurement, pestilence, et chagrin des proches, vous appelez ça «La vie! Oh! La vie!» ? Oui, c'est encore la vie, l'envers de la vie, un envers insupportable, et c'est là que les gens demandent, bien raisonnablement, leur laissez-passer pour l'au-delà.

Si un chien était dans un tel état, on l'achèverait par compassion. C'est tout ce que je demande: un peu de compassion. SVP, un peu de pitié et de compassion. SVP. SVP. SVP!!!

J'espère que ces réflexions auront pu vous éclairer sur ce grave enjeu qu'est l'euthanasie et sur sa nécessité, bien encadrée, dans une société humaine et civilisée, libérée des tabous. Merci.

Claire Morissette,

\section{Note}

1. Frontières remercie Francine Lalonde, Guy Lamarche et l'Association québécoise pour le droit de mourir dans la dignité qui nous ont autorisés à publier ce texte qui figure parmi les «Textes fondateurs » disponibles sur le site Web de l'association à l'adresse suivante: <www.aqdmd.qc.ca/texte.fondateur. Morissette.html $>$. 